\title{
Molecular Properties of Interfaces Relevant for Clathrate Hydrate Agglomeration
}

\author{
Alberto Striolo* and Anh Phan \\ University College London \\ Department of Chemical Engineering \\ Torrington Place \\ London, WC1E 7JE \\ UK
}

\section{Matthew R. Walsh}

Chevron Exploration and Production

Covington, LA 70433

USA

\begin{abstract}
Clathrate hydrates of natural gases show promise for energy in regions traditionally poor of fossil fuels, suggest the possibility of mitigating the atmospheric impact of hydrocarbon consumption via $\mathrm{CO}_{2}$ sequestration, could advance high-tech applications to address global challenges, including the water-energy nexus, and can lead to significant economic loss and potential safety hazards when they form in oil and gas pipelines. This focused review is motivated by several recent contributions, which demonstrate how atomistic and coarsegrained simulations have become a useful tool. The examples provided suggest the time is right to take advantage of the enhanced understanding provided by simulations, to couple them with experiments that could help design new chemicals for managing hydrate formation in pipelines, for designing chemical processes and additives to advance high-tech applications such as water desalination and natural gas storage, and perhaps also for the production of methane from geological hydrate deposits with simultaneous carbon dioxide sequestration.
\end{abstract}

Keywords: Flow assurance, surfactants, nucleation and growth, molecular design

* Author to whom all correspondence should be addressed:

E.: a.striolo@ucl.ac.uk

P.: +44 (0) 2076793826 


\section{Introduction}

Clathrate hydrates of natural gases form because of a balance between guest-host (e.g., methane-water) dispersive interactions and hydrogen bonds among water molecules [1]. Several clathrate structures are known, their relative stability depending on the guest and on pressure-temperature conditions [2]. The most common crystalline hydrate structures are sl, sll, and $\mathrm{sH}$, with $\mathrm{sl}$ and sll the structures most often formed by natural gases. Geological deposits of natural gas hydrates [3] could contain more hydrocarbons than humankind has used so far; geological hydrates deposits could also be used to sequester carbon dioxide [4]. Clathrate hydrates could be designed to store natural gas at near-ambient conditions [5]. For the latter application, Kumar et al. [5] quantified the properties of mixed methanetetrahydrofuran hydrates, which show thermodynamic and kinetic advantages towards the development of natural gas storage technologies at near atmospheric pressures. They also reported in-situ Raman spectroscopy and powder X-ray diffraction data, useful for better understanding hydrates at the molecular level.

On the other hand, the energy sector invests heavily to prevent the agglomeration of hydrates in oil-and-gas pipelines [6]. The related technologies are traditionally developed via an arsenal of experimental techniques $[7,8]$. We believe that computational tools can positively impact this field, and some evidence in support of this hypothesis is reviewed herein.

In what follows, after a summary on the most common strategies to manage hydrates, we review recent simulations implemented to better manage hydrates and suggest some research topics that we believe are timely. For background on molecular simulations, we refer to textbooks [9]. The rationale is that once the interactions between molecules are known, structure, thermodynamic and possibly kinetic properties of reactive solid/fluid mixtures can be predicted, e.g., by integrating the equations of motion for the molecules as the system evolves within selected constraints. Uncertainties in force fields, algorithms sometimes inadequate to sample, e.g., rare events, and limited computational power are typical obstacles, but progress is promising $[10,11]$.

\section{Managing Hydrates: Thermodynamic vs. Low Dosage Inhibitors}

When natural gas and water molecules are both present at high pressure and low temperature, hydrates might form. The formation of hydrate plugs involves several processes, including hydrate nucleation, growth and agglomeration [12]. Large hydrate plugs could block pipelines and subsea flowlines, causing safety, environmental and economic threats [6]. To mitigate these risks, hydrates are prevented or managed commercially primarily via three approaches:

1. Engineering design: it is possible to prevent the produced fluid from entering the hydrate-stability region during flow, via modelling the transport of multi-phase systems [13] and determining required insulation thickness, pipeline size and possible subsea pumping needs. Because the composition and rate of the produced hydrocarbons can change as wells age (i.e., lower flow overcoming the added specific heat of higher water content, with the higher water content raising the risk of plug formation), the fluid could still enter the hydrate stability region and form blockages during flow late in field life [14]. Also, due to inevitable temporary shut-ins (e.g., for compliance), the system will enter the hydrate zone during transients throughout the life of the development. One engineering method to combat these transient excursions into the hydrate stability zone is the use of direct electrical heating of the pipeline [15], applied before initiation of flow. 
2. Thermodynamic hydrate inhibitors (THIs): THIs (e.g., methanol) shift the hydrate stability conditions to higher pressures and lower temperatures $[16,17]$. Combining THIs with pipeline design, it is possible to ensure that the system does not enter the hydrate stability region during planned temporary transients by treating for example uninsulated wellheads and jumpers before shut-in and restarting the system before the insulated flowline reaches the hydrate stability zone. Equations of state are developed to predict the hydrate stability region for complex systems [18]. Sa et al. [16] reviewed salient data and identified reliable datasets via a thermodynamic consistency analysis. However, it should be noted that THIs are only effective at high weight fraction with respect to the water content (20-50\%) [19].

3. Low dosage hydrate inhibitors (LDHIs): LDHIs are effective at $0.5-3.0 \mathrm{wt} \%$ of the water content, but some LDHIs show low biodegradability [19], and their mode of action is not fully understood. There are two main LDHIs classes: kinetic hydrate inhibitors (KHIs) and anti-agglomerants (AAs). Kelland provided a historical perspective on LDHIs [19], including early simulations [20]. While KHIs are generally polymers, AAs tend to be surfactants [21]. KHIs should delay hydrates' nucleation and slow their growth while AAs are expected to prevent their agglomeration. In some cases, AAs facilitate hydrate formation, and in all cases an effective and properly-dosed AA will yield a hydrate slurry that can be transported. Oil properties (composition, density, viscosity, emulsion tendency) and the amount of gas present (quantified by 'gas-in-oil' ratio) affect LDHIs' performance [22]; increasing salt concentration usually enhances AAs performance [23]. Small changes in LDHIs' molecular structure alter dramatically their performance [21]. It is customary to conduct systematic experiments to identify optimal LDHIs dosage and composition, and research is ongoing to identify LDHIs that are effective, environmentally benign and affordable [19]. Most research in this area to date has been experimental, with simulations a recent addition to the body of research, as discussed below. The simulations mechanistic component will couple well with the established "pass/fail" criteria of experiments to accelerate research.

\section{Recent Simulations}

Recent successes suggest that molecular simulations could help understanding the mechanisms, eventually leading to improved hydrate management. We structure this discussion around a few relevant topics:

Nucleation. Brute-force molecular dynamics (MD) simulations can yield nucleation [24]. In brute-force MD simulations, a system is prepared in the fluid phase, and the equations of motion are integrated. The fluid molecules yield solid structures after a 'critical nucleus' is formed. Multiple groups have studied hydrate formation with varying molecular models, ensembles and thermodynamic conditions [25]. The results from brute force simulations suggest hydrate formation occurs via a cooperative adsorption and growth mechanism between guest and host, and (despite some variability in resulting crystallinity) that the resulting post-critical solid structure at high driving force is generally amorphous while the resulting solid structure at lower driving forces is more crystalline, suggesting multiple drivingforce-dependent pathways [26-31]. Alternative methods to the brute force technique include the Forward Flux method [32], and metadynamics with restrained MD [33], employed by Lauricella et al., who suggested the main "pre-simulation" theories for the hydrate nucleation mechanism may all occur. Zhang et al. [34] noted that the inception time for the formation of 
sl hydrates is strongly correlated with the self-diffusion coefficient of the guest molecules. In these simulation methods, specialised algorithms are implemented to 'guide' the system trajectories towards hydrate formation, rather than spending extensive simulation times sampling fluid structures. It should be noted that impurities could provide nucleating agents $[35,36]$.

Understanding nucleation could lead to new KHIs. Poon et al. [37] e.g., suggested design principles starting from the classic nucleation theory. Recent nucleation studies couple order parameters, defined using short-ranged correlations among molecules, with enhanced sampling techniques, e.g., metadynamics, to observe the rare events leading to nucleation. Gobbo et al. recently proposed a systematic approach to develop order parameters that quantify the difference between distributed properties (e.g., long-ranged correlations) of a system and those of a crystalline structure [38]. This approach promises to be applicable to complex systems such as those in which hydrates form in pipelines.

Growth and Dissociation. Michalis et al. [39] simulated the growth of $\mathrm{CO}_{2}$ and $\mathrm{CH}_{4}$ hydrates as a function of temperature and pressure, achieving excellent agreement with experiments. In these simulations, a hydrate nucleus is surrounded by water and exposed to hydrocarbons. As the MD simulations progress, the hydrate grows. This approach could be used to quantify how chemical additives/guests, e.g., tetrahydrofuran [5], affect hydrate growth rate and stability. Bui et al. [40], e.g., simulated hydrate growth in the presence of AAs (see Figure 1). Those simulations combined brute force MD with metadynamics. The results suggest that AAs could control methane transport across the hydrocarbon-water interface, as well as act as templating agents, structuring water molecules to enhance hydrate growth rate. The barrier to methane transport was observed when the AAs, in combination with hydrocarbon chains from the hydrocarbon phase, formed an ordered interfacial film, within which the density of methane was nearly zero. More on this is discussed in the section "AAs at Water-Hydrocarbon Interfaces". The AAs simulated are shown in panel (B) of Figure 1. These AAs have 3 hydrophobic tails: two $R_{1} S$ are equal and generally long, while $R_{2}$ tends to be short. In Bui et al.'s simulations, $R_{2}$ was found to preferentially reside near the aqueous phase, while $R_{1} S$ extended towards the hydrocarbons. In some cases, $R_{2}$ seemed to favour hydrate growth by templating water molecules in cage-like structures that were quickly occupied by methane. Investigating hydrate dissociation can also help us understand and design systems involving thermodynamic cycles such as refrigeration, and potentially the production of hydrocarbons from or sequestering of $\mathrm{CO}_{2}$ in biogenic and thermogenic hydrate deposits. In general, these simulations start from a hydrate seed in equilibrium with a hydrocarbon phase, and the simulation conditions (e.g., the temperature) are changed (e.g., increased) outside the region of hydrate stability. As the simulations progress, the hydrate seeds dissociate. Ghaani and English recently quantified the effect of electromagnetic fields on the dissociation rate of propane hydrates [41]. The results, summarised in Figure 2, could identify processes and chemicals that promote fast dissociation of hydrate plugs (although heat-transfer limitations may prevail in practice). Explicitly, in the absence of the electric field, the melting temperature of the propane hydrate considered by Ghaani and English is estimated in $266 \pm 3 \mathrm{~K}$; the observed melting temperature negligibly changed for the fields less than $0.07 \mathrm{~V} / \mathrm{nm}$; however, increasing the applied static electric field to $0.7 \mathrm{~V} / \mathrm{nm}$ caused the hydrates to melt at $250 \mathrm{~K}$ (top panel of Figure 2). The sequence of simulation snapshots shown on the bottom panel of Figure 2 suggests that when the temperature is $563 \mathrm{~K}$ and the applied static electric field is $0.007 \mathrm{~V} / \mathrm{nm}$, the hydrate cages become highly distorted before they dissociate, and 
that the propane molecules originally trapped as a guest within these cages form nanobubbles dispersed within the system.



Figure 1. Atomistic simulations of hydrate growth in the presence of AAs. In panel (A) the simulated system is shown, where are highlighted the crystal hydrate seed, the film of liquid

water, the anti-agglomerants adsorbed at the water-hydrocarbon interface, and the hydrocarbon phase, composed of dodecane and methane. In panel (B) the schematic representation of the anti-agglomerants considered by Bui et al. [40] is provided. In panel

(C) a schematic is provided for the growth of the hydrate in the presence of AAs: the simulations start with the system shown on the left and are concluded when all the free water has been converted in hydrate (right). In panel (D) the results are shown in terms of the amount of water that is within the hydrates as opposed to liquid. As the simulations progress, liquid water becomes hydrate. The results in black are obtained without AAs, while the other three lines are obtained in the presence of three different AAs. Notably, for the results in red, the respective AAs were able to prevent methane transport across the hydrocarbon-water interface, which delayed hydrate growth at the conditions tested. In panel $(E)$ results are shown for the free energy landscape monitored during hydrate growth in the presence of AAs. CV1 and CV2 are two 'collective variables' (i.e., one represents the position of a surfactant tail, and the other its coordination number with surrounding methane molecules), useful to monitor the properties of the system. The results show that one the AAs tail highlighted as $R_{2}$ in panel (B) actively promotes hydrate growth. Adapted with permission from Ref. [40]. Copyright 2018 American Chemical Society. 


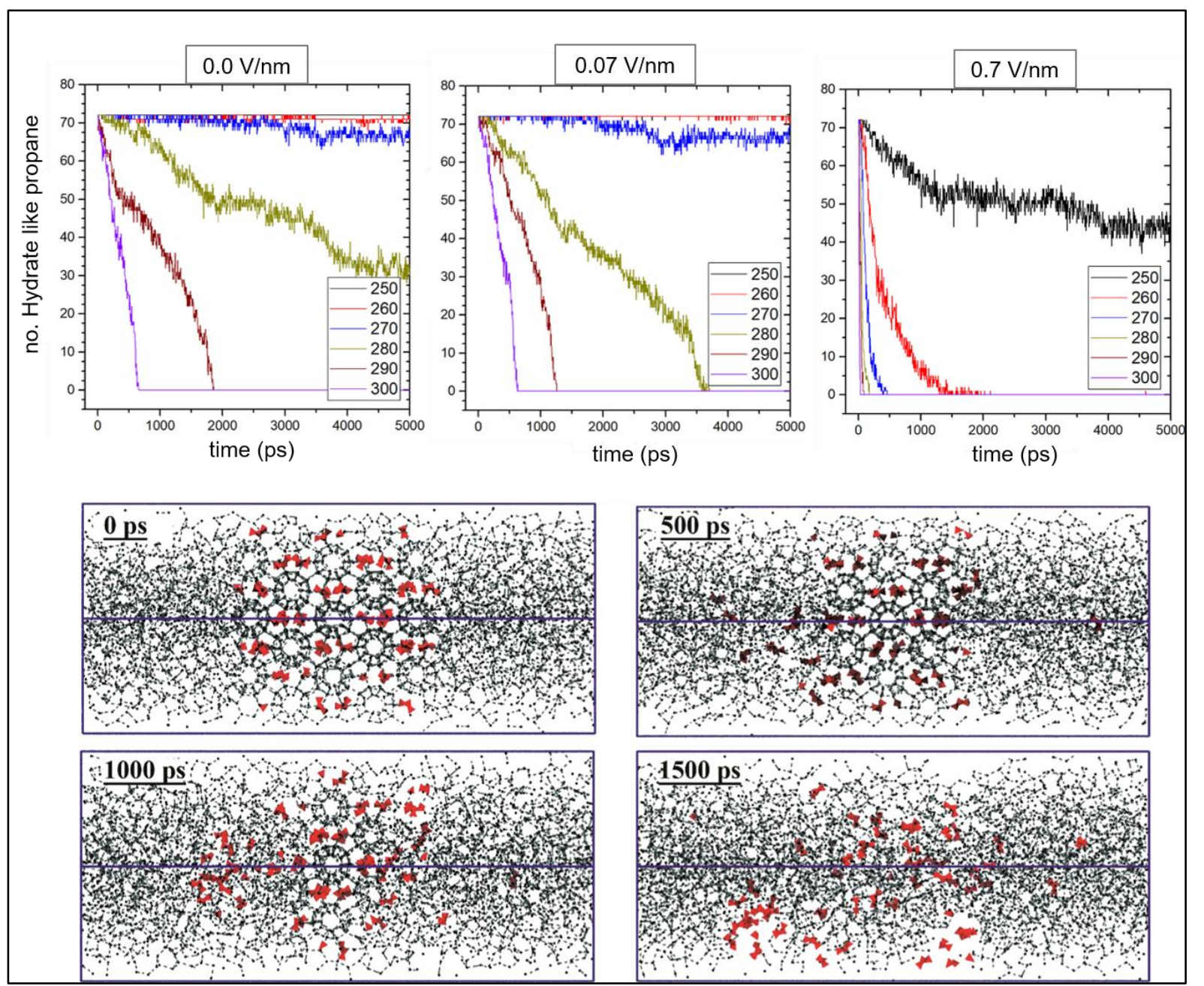

Figure 2. Representative results for simulation studies on hydrate dissociation. The simulations are conducted for the sll propane hydrate, whose equilibrium melting point was estimated to be $266 \pm 3 \mathrm{~K}$. In the top row panels of this figure, the number of hydrate-like propane molecules is plotted as a function of simulation time. The simulations are conducted at various temperatures, which are reported in the legends in $\mathrm{K}$ units. When there is no external electric field applied (left) the results show that the hydrate dissociates at a faster rate as the temperature increases above $270 \mathrm{~K}$. In the top middle and right panels, the results show that as a static external electric field is applied at increasing voltages (from 0.07 to $0.7 \mathrm{~V} / \mathrm{nm}$ ), the melting temperature decreases and the dissociation rate increases. In

the bottom panels the simulation snapshots illustrate the mechanism of hydrate dissociation. These simulations were conducted $563 \mathrm{~K}$ and an applied $0.007 \mathrm{~V} / \mathrm{nm}$ static electric field. The insets indicate the length of the simulations. The red molecules represent propane, initially within the hydrate crystal. The dotted lines identify the hydrogen bonds between water molecules, indicated as black spheres placed in correspondence of their oxygen atoms. The sequence of snapshots shows that hydrates cages deform and distort before dissociation. Once free from the hydrate, propane molecules yield nano-bubbles. Figure adapted with permission from Ref. [41]. Copyright 2018 American Chemical Society. 
KHIs and AAs at Water-Hydrate Interfaces. Because the LDHIs' effectiveness frequently decreases as the water content increases, Anderson et al. [42] calculated the adsorption free energy for monomers representing widely-adopted KHIs at the hydrate-water interface. These calculations are typically conducted via quantifying the 'potential of mean forces': the average force between the hydrate surface and the monomers is integrated as a function of the distance. Anderson et al. reported a positive correlation between adsorption energy and experimental performance. In other words, the monomers of those KHIs that were known to be effective in practical applications were found to be strongly attracted to the hydrate surface (more negative potential of mean forces compared to values far from the surface). Building from that work, several AAs were simulated at the hydrate-water interface $[43,44]$. Conducted for one surfactant at the interface, these atomistic studies revealed preferential adsorption sites, adsorption mechanisms on the hydrate surfaces, and AAs conformational properties. For example, Mehrabian et al. [44] quantified the free energy of adsorption for $n$ dodecyl-tri(n-butyl)-ammonium chloride at the sll hydrate - water interface at $277 \mathrm{~K}$ and 100 bar and provided molecular explanations for the experimental observation that increasing salt content enhances AAs performance (see Figure 3) [23]. As the $\mathrm{NaCl}$ concentration increased from 0 to 3.5 and $10 \mathrm{wt} \%$ the free energy of adsorption decreased by 1.9 and 4.3 $\mathrm{kcal} / \mathrm{mol}$, respectively. Mehrabian et al. [45] further quantified the effect of tail length on adsorption, systematically varying the alkyl tail length from 8 to 16 carbons. They reported more effective adsorption when the tail has 12 carbon atoms, in qualitative agreement with experimental data for AAs performance [21]. In a different contribution, Jimenez Angeles and Firoozabadi [43] simulated one hydrocarbon chain, one non-ionic and one ionic surfactant, showing a positive correlation between hydration water structure and adsorption energy. Because salts affect the hydration structure on hydrophobic tails, they control the adsorption of non-ionic surfactants on hydrate-water interfaces.

These simulations suggest that the stronger the AAs adsorption at the water-hydrate interface, the better their performance. Should this correlation hold quantitatively, simple simulations could identify promising AAs. Perhaps, however, other interfaces and cooperative phenomena (e.g., self-assembly) are also important for determining the performance of AAs. The effect of AAs on hydrate-hydrate effective interactions in water should also be quantified. 


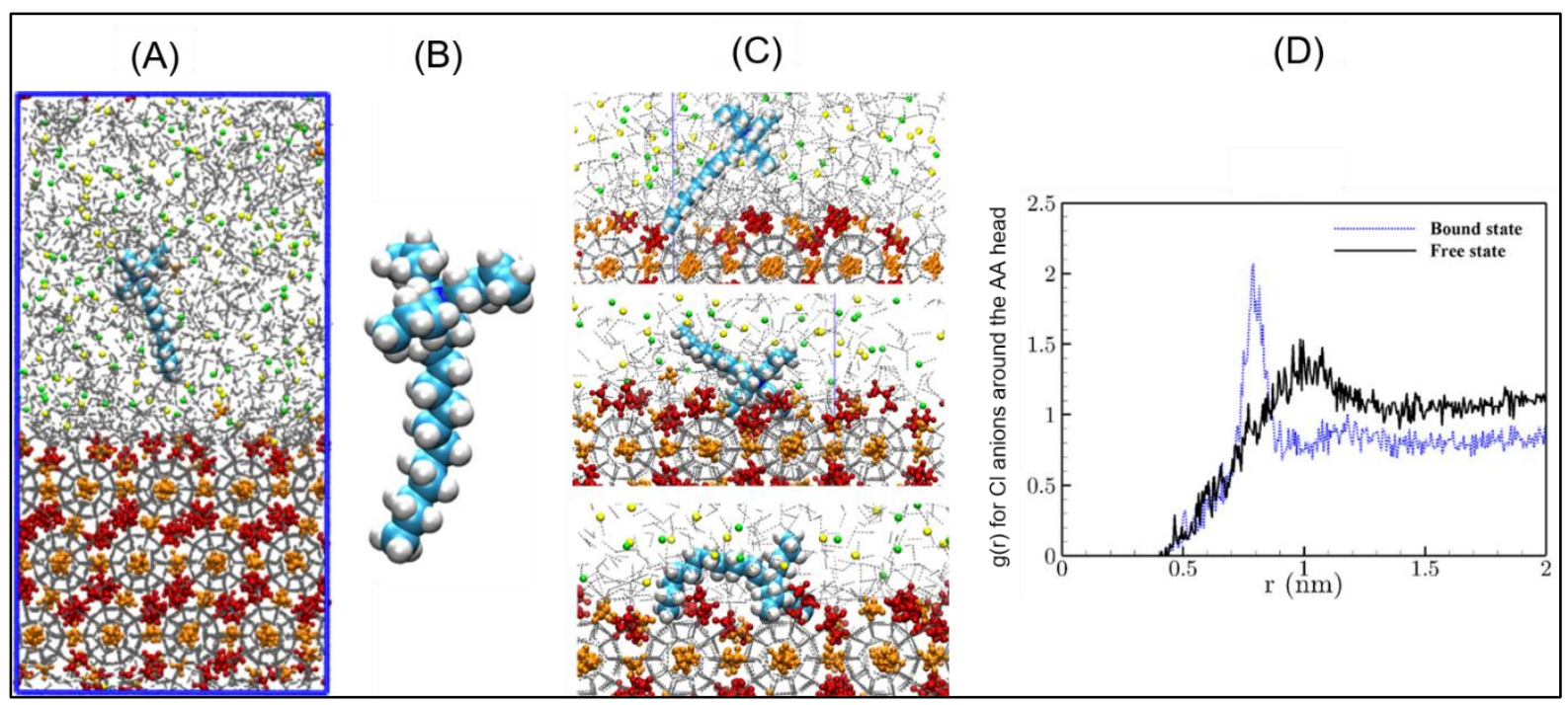

Figure 3. Representative simulation results for AAs at the water - sll hydrate interface.

These simulations are conducted for a single n-dodecyl-tri(n-butyl-ammonium) chloride AA, which adsorbs at the solid liquid interface. In panel (A) a representation for the simulated

system is provided. Water molecules are shown in grey; within the hydrate structure, methane in shown in yellow and propane in red; within the aqueous phase green and yellow spheres represent sodium and chloride ions, respectively. In panel (B) the molecular structure of the AA simulated is shown. In this AA three short alkyl chains construct the head (the tripod), and one long straight hydrocarbon chain is the tail. AAs simulated at the sll hydrate - water interfaces. In panel (C) the three possible adsorbed configurations are shown. From top to bottom, these are: only the tail is adsorbed, only the head is adsorbed, and both tail and head are adsorbed, forming a molecular bridge. In panel (D) the results for the radial distribution function between chloride ions and AAs head groups are reproduced. The results obtained in bulk water are compared to those obtained when the AA is adsorbed at the hydrate-water interface. The results show that the first peak in the radial distribution function becomes pronounced upon adsorption. This figure is adapted with permission from Ref. [44]. Copyright 2018 American Chemical Society.

AAs at Water-Hydrocarbon Interfaces. Dieker et al. [46] introduced the micromechanical force apparatus to measure interactions between hydrate particles and water droplets in oils. These experiments may reproduce low-to-medium water content conditions, at which AAs should adsorb at the hydrate/water - hydrocarbon interface. Bui et al. [47] conducted atomistic equilibrium MD simulations for AAs on these interfaces. The AAs contained multiple hydrophobic tails and an extensive hydrophilic head with one quaternary ammonium ion (see panel (B) in Figure 1). For these AAs, small tail length variations yield large changes in performance. For example, when the linear chain identified as $R_{2}$ in Figure 1 contains 4 carbon atoms, the experimental performance is reported as 'good' when $R_{1}$ is a linear chain of 12 carbon atoms, but 'poor' when $\mathrm{R}_{1}$ contains 8 carbons [40]. The molecular mechanisms responsible for these changes in experimental performance are not fully understood, but simulations could help. Because atomistic MD can only sample hundreds of nanoseconds, much shorter than the microseconds required by surfactants to exchange between micelles, Bui et al. [47] prepared interfaces at different AAs coverage. The AAs effective in preventing hydrate plugs formation experimentally, yielded ordered films at sufficiently high surface 
density. The hydrocarbon molecules were essential for this phenomenon. When the AAs $R_{1}$ tails length (12 carbon atoms) was comparable to that of the oil (n-dodecane), dodecane molecules intercalated the interfacial film, yielding compact and ordered structures. Because the density of methane within such interfacial films was negligible, Bui et al. [47] suggested that the AAs they considered could prevent hydrate plug formation, in part, causing an interfacial barrier that prevent both water and methane from reaching the hydrate particle. Sicard et al. [48] quantified the strong free energy barrier encountered by methane when it travels across such interfaces. For these calculations, enhanced sampling techniques including metadynamics, umbrella sampling, and adiabatic biased MD were implemented. The results were interpreted within the framework of the transition state theory.

Simulation results for AAs at interfaces strongly suggest that the mechanisms of action of different AAs could vary widely. Perhaps the system compositions (i.e., water chemistry, oil composition, temperature, pressure) and impurities could determine the mechanism that is dominant in a specific situation. Thus, careful studies are required for diverse AAs families, when possible coupling macroscopic experiments (e.g., rocking cell) with microscopic ones using the micromechanical force apparatus and perhaps via tip-enhanced Raman spectroscopy. Such a synergistic combination of simulations and experiments at multiple length scales could provide sufficient validation and help the community design new LDHIs. Similar approaches have been very effective for designing promising new drugs.

Agglomeration and Ripening. Inspired by micromechanical force experiments [46], Phan et al. [49] simulated how water droplets coalesce on hydrates in the presence of AAs. In these MD simulations one water nano-droplet and one hydrate particle are prepared within a hydrocarbon phase. Within the framework of 'steered MD', the nano-droplet is gently pushed towards the hydrate particle, and the force applied is recorded as a function of the distance. Two are the main limitations: the droplets/hydrate particles are much smaller than experimental ones, and the water droplet is dragged at unrealistically-high velocities. Despite these limitations, the results [49] demonstrated that when not enough AAs are present, coalescence is spontaneous and irreversible once water molecules form a bridge across the interfacial AAs, and that, for AAs with quaternary ammonium ions, a tripod of 4 carbons promotes adsorption on the hydrate, while increasing the tail length does not necessarily enhance performance.

One mechanism that could affect hydrate plug formation, but is difficult to probe, is Ostwald ripening. Khedr and Striolo [50] using coarse-grained simulations, successfully reproduced literature data for benzene droplets dispersed in water. In coarse-grained simulations one does not describe each atom in a system, but rather describes several molecules as a single 'bead'. This allows for significant savings in computing time, at the cost of not including atomic-level details in the results. Using the algorithm proposed by Khedr and Striolo, one could design AAs that can decrease Ostwald ripening rate by decreasing the interfacial tension between water and oil, and by causing an additional rate-limiting step in the process of water diffusion from a water droplet to a growing hydrate, a possibility suggested by the results reported by Bui et al. [47]. Could experiments and simulations probe these mechanisms, perhaps via multi-scale approaches?

The Whole Picture. The paragraphs above consider individual mechanisms by which LDHIs affect hydrates. To assemble the whole picture, one could implement stochastic models, e.g., 
kinetic Monte Carlo (kMC), which has been recently implemented to quantify nanoparticle formation [51] and fluid transport across pore networks [52]. Describing stochastically the formation of hydrate plugs requires, e.g., free energy barriers concerning nucleation, growth, dissociation and agglomeration of hydrates at different conditions. One kMC advantage consists in its relatively low computational cost, but the accuracy of the results depends strongly on the accuracy of the input parameters that describe the probabilities of the various events, which are likely to change with system composition and thermodynamic conditions.

Alternatively, one could simulate large systems using coarse-grained models. These approaches strongly depend on the accuracy of the force fields and on the size of the systems being simulated, with larger systems expected to provide more reliable results because they can account for multiple phenomena occurring simultaneously; benchmarking against both experiments and atomistic simulations is necessary before simulating complex systems using coarse-grained approaches. Naullage et al. [53], e.g., recently probed how surfactants could act as AAs using a coarse-grained simulation. The results reinforce the atomistic simulations conclusions, while providing additional insights. They found that dodecanol adsorbs on the interface between sl and sll hydrates and the hydrocarbon n-pentane. At 275K and 100 bar, the enthalpic contribution to the free energy of adsorption for dodecanol is $-12.9 \mathrm{kcal} / \mathrm{mol}$, while the entropic contribution is $+4.4 \mathrm{kcal} / \mathrm{mol}$ (unfavourable). Dodecanol was highly mobile at the hydrate-oil interface, despite the strong adsorption. Surface diffusion is governed by hopping events, which slow down the diffusion compared to that observed at oil-water interfaces. The atomistic simulations by Phan et al. [49] also suggested that the surface diffusion of AAs at hydrate-oil interfaces strongly depends on the features of the surfactant headgroup. Also consistent with atomistic simulations [47], Nullage et al. [53] showed that dodecane and dodecanol co-adsorb at the hydrate-dodecane interface. For example, at $41 \%$ of the full dodecanol monolayer, dodecane intercalation causes the interfacial dodecanoldodecane film to yield an interfacial layer with density 95\% that of the crystal. These results are consistent with the simulations of Bui et al. [47], with experimental surface tension data in the presence of cetyltrimetylammonium bromide [54], and other experiments [55]. Also in agreement with atomistic simulations [47], ordered films were not obtained when the surfactant tail was too short (i.e., hexanol) even in the presence of hexane. In agreement with experiments [56], the simulations showed that surfactants adsorption changes the nature of the hydrate interface (Figure 4). By quantifying the simulated contact angles, Naullage et al. [53] estimated the surface free energy of the clathrate-dodecane interface as $76.9 \pm 2 \mathrm{~mJ} \mathrm{~m}^{-2}$. The snapshots of Figure 4 show the contact angle for water reduces from $180^{\circ}$ in dodecane to $88 \pm 2^{\circ}$ in vacuum: dispersive interactions between water and hydrocarbons stabilize the water droplet. Naullage et al. [53] also quantified the free energy landscape encountered by a water droplet as it transports across the interfacial surfactant film. The surfactants only provide a kinetic barrier, and as soon as a channel forms across the surfactant film, coalescence is irreversible. The results, consistent with the simulations by Phan et al. [49] as well as with experiments [19], suggest that increasing the adhesion between surfactants and clathrate could enhance AAs performance.

Coarse-grained simulations promise to address simultaneously a number of phenomena that occur within the pipeline. Because many commercial AAs are quaternary ammonium surfactants $[19,21]$, these simulations should be repeated when models consistent with coarse-grained water are developed for ionic surfactants. Perhaps coarse-grained force fields developed to study lipid bilayers could be helpful. One future challenge will be coupling the 
level of details accessible via equilibrium simulations with fluid dynamics multi-phase studies. Perhaps, using kMC, it will be possible to extract effective parameters from the simulations to be used as input for fluid dynamics models.

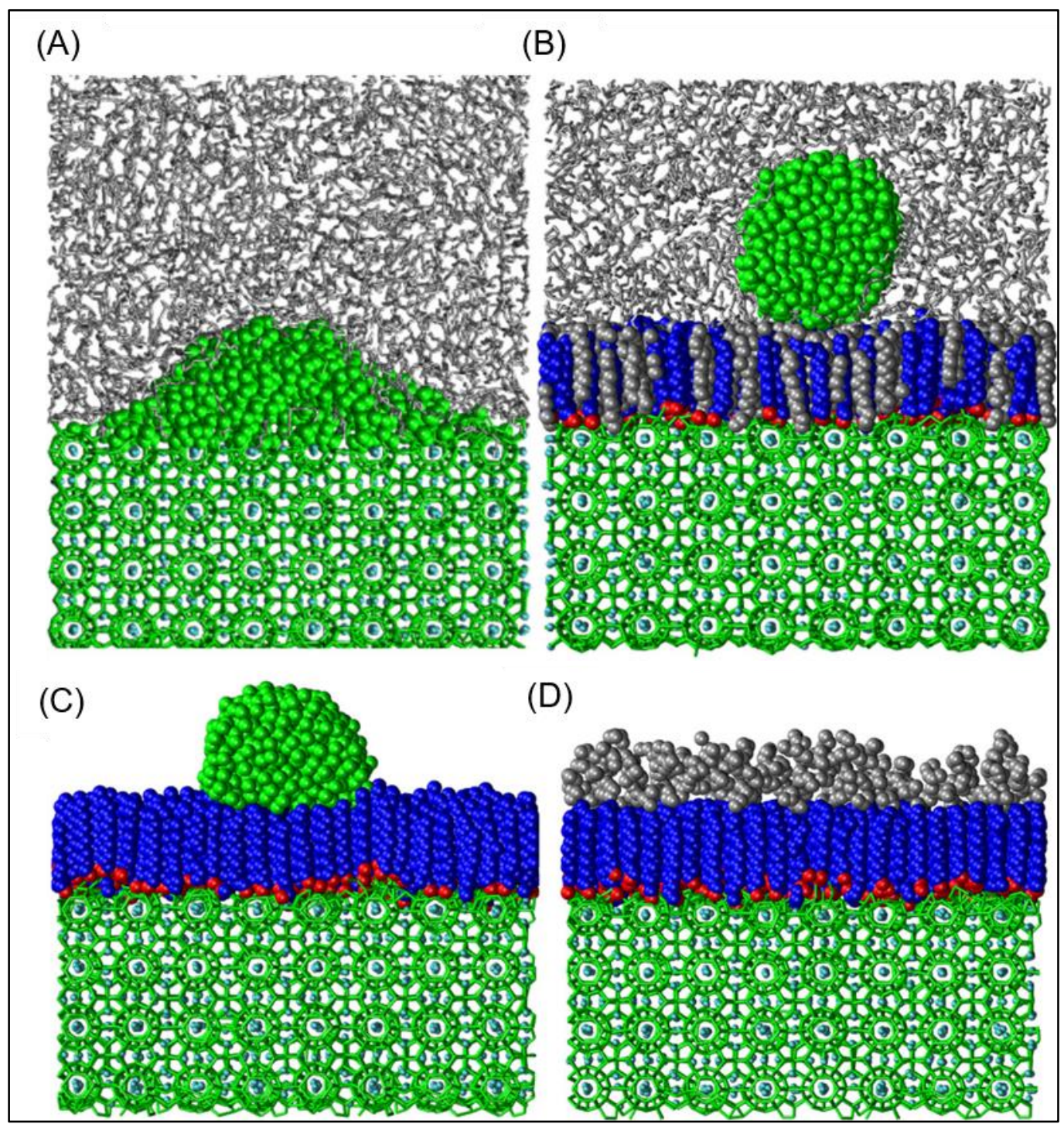

Figure 4. Simulation snapshots representing the interfacial properties of the sl hydrate filled with methane (green crystal). The blue-red molecules represent the dodecanol surfactants,

with the red spheres representing the hydrophilic head groups and the blue ones the hydrophobic components of the tail groups. Green spheres represent water molecules within a mono-atomic coarse-grained model, and grey molecules represent hydrocarbons.

The simulations were conducted at $277 \mathrm{~K}$ and 100 bar, at varying surface coverage of surfactant, from zero (panel A) to $4.43 \mathrm{~nm}^{-2}$ (panel B) to $5.22 \mathrm{~nm}^{-2}$ (panels $C$ and D). In panel $(A)$ the results show that water wets the hydrate-oil interface, while in panel (B) it is evident that the interface has become hydrophobic because of the presence of the mixed interfacial

film composed of surfactants and intercalated oil molecules. In panels (C) and (D) the wetting properties for water and dodecane are quantified at the hydrate covered by surfactants, respectively. Reproduced from Naullage et al. [53]. 


\section{Future Directions}

The long-term ambition of computational chemistry is to design - in silico - new compounds effective for practical applications. Achieving this ambition requires coupling state-of-the-art simulations with advanced experiments, to ensure that the force fields and the algorithms are reliable, and to ensure that the interpretation of the experimental results is realistic. At the moment, such synergism is hampered by the lack of extensive experimental data that directly probe the interfaces of interest at the molecular resolution, for example via advanced techniques such as tip-enhanced Raman spectroscopy and NMR [57], by the limited time and length scales accessible via simulations, and by the approximations built in the development of atomistic and coarse-grained simulation force fields. Micromechanical force measurements yield meso-scale information, including, e.g., the contact angle between water and hydrate in the presence of AAs. This information is a valuable stepping stone connecting molecular-scale data and macroscopic observations, which include rocking cell experiments and flow loops tests. Macroscopic experiments are needed before new LDHIs are introduced in the field, but they only provide qualitative comparison for simulations. When a full multiscale simulation approach is developed, inclusive of atomistic and coarse grained details, stochastic kMC modelling of hydrate agglomeration and growth, as well as multi-phase fluid dynamics calculation of fluid transport in flow loops, a true synergism will be achieved between modelling and experiments at all relevant length and time scales, which is ultimately required to manage the risk of hydrate formation in pipelines, and may facilitate the ability to control hydrates to meet other important challenges.

\section{Acknowledgments}

AS acknowledges financial support from the EPSRC, under grant number EP/N007123/1, from The Royal Society, under the International Exchange grant number IE160820, and from Halliburton, who partially supported one Ph.D. studentship focused on AAs. Intellectual contributions from his group members, in particular Dr Tai Bui, Dr Anh Phan, Dr Francois Sicard and Ms Abeer Khedr are highly appreciated.

\section{Conflicts of Interests}

The Authors declare no conflicts of interests.

\section{References and Notes}

1. Koh CA: Towards a fundamental understanding of natural gas hydrates. Chem Soc Rev 2002, 31:157-167.

2. Fleischer EB, Janda KC: Prediction of Clathrate Structure Type and Guest Position by Molecular Mechanics. J Phys Chem A 2013, 117:4001-4010.

3. Makogon YF: Natural gas hydrates - A promising source of energy. J Nat Gas Sci Eng 2010, 2:49-59.

4. Schoderbek D, Martin KL, Howard J, Silpngarmlert S, Hester K: North Slope Hydrate Fieldtrial: $\mathbf{C O}_{2} / \mathbf{C H}_{4}$ Exchange. OTC 23725, Arctic Technology Conference, Houston, TX, USA, 3-5 December 2012.

5. Kumar A, Veluswamy HP, Linga P, Kumar R: Molecular level investigations and stability analysis of mixed methane-tetrahydrofuran hydrates: Implications to energy storage. Fuel 2019, 236:1505-1511.

This contribution is important for both practical and fundamental reasons. From the practical point of view, the Authors quantify the properties of mixed methane-tetrahydrofuran hydrates, which show thermodynamic and kinetic advantages towards the development of natural gas storage technologies at near atmospheric 
pressures. From the fundamental point of view, in-situ Raman spectroscopy and powder X-ray diffraction provide useful data for the development of advanced understanding on this subject.

6. Zerpa LE, Salager JL, Koh CA, Sloan ED, Sum AK: Surface Chemistry and Gas Hydrates in Flow Assurance. Ind Eng Chem Res 2011, 50:188-197.

7. Bagherzadeh SA, Alavi S, Ripmeestera JA, Englezos P: Why ice-binding type I antifreeze protein acts as a gas hydrate crystal inhibitor. Phys Chem Chem Phys 2015, 17:9984-9990.

8. Morrissy SA, McKenzie AJ, Graham BF, Johns ML, May EF, Aman ZM: Reduction of Clathrate Hydrate Film Growth Rate by Naturally Occurring Surface Active Components. Energ Fuel 2017, 31:5798-5805.

9. Allen MP, Tildesley DJ: Computer Simulation of Liquids. Oxford, UK: Oxford University Press; 2004.

10. Pfaendtner J, Snurr RQ, Van Speybroeck V: Frontiers of chemical engineering: Molecular modeling. Curr Opin Chem Eng 2019, 23:A1-A2.

11. Striolo A: Studying surfactants adsorption on heterogeneous substrates. Curr Opin Chem Eng 2019, 23:115-122.

12. Metaxas PJ, Lim VWS, Booth C, Zhen J, Stanwix PL, Johns ML, Aman ZM, Haandrikman G, Crosby D, May EF: Gas hydrate formation probability distributions: Induction times, rates of nucleation and growth. Fuel 2019, 252:448-457.

These experimental results suggest the need to improve theoretical models of hydrate formation, as the measured nucleation rates are much slower than those predicted using the classical nucleation theory.

13. Charlton TB, Di Lorenzo M, Zerpa LE, Koh CA, Johns ML, May EF, Aman ZM: Simulating Hydrate Growth and Transport Behavior in Gas-Dominant Flow. Energ Fuel 2018, 32:1012-1023.

14. Mehta AP, Hebert PB, Cadena ER, Weatherman JP: Fulfilling the Promise of Low Dosage Hydrate Inhibitors: Journey from Academic Curiosity to Successful Field Implementation. OTC 14057, Offshore Technology Conference, Houston, TX, USA, 6-9 May 2002.

15. Boxall JA, Ng SH, Aman ZM, Norris BWE, Hughes TJ, Joannou K, May EF: Hydrate Plug Dissociation via Active Heating: Uniform Heating and a Simple Predictive Model. Energ Fuel 2016, 30:9275-9284.

16. Hu Y, Sa JH, Lee BR, Sum AK: Universal Correlation for Gas Hydrates Suppression Temperature of Inhibited Systems: III. Salts and Organic Inhibitors. AIChE J 2018, 64:4097-4109.

17. Chaudhari $P$, Zerpa $L E$, Sum AK: A correlation to quantify hydrate plugging risk in oil and gas production pipelines based on hydrate transportability parameters. J Nat Gas Sci Eng 2018, 58:152-161.

18. Sa JH, Hu Y, Sum AK: Assessing thermodynamic consistency of gas hydrates phase equilibrium data for inhibited systems. Fluid Phase Equilibr 2018, 473:294-299.

This paper reviews a large amount of experimental data for the phase equilibria of hydrates in the presence of different inhibitors, and it identifies reliable datasets via a thermodynamic consistency analysis.

19. Kelland MA: History of the development of low dosage hydrate inhibitors. Energ Fuel 2006, 20:825-847.

20. Carver TJ, Drew MGB, Rodger PM: Inhibition of Crystal-Growth in Methane Hydrate. J Chem Soc Faraday $T$ 1995, 91:3449-3460.

21. Chua PC, Kelland MA: Study of the Gas Hydrate Anti-agglomerant Performance of a Series of $n$-Alkyl-tri(nbutyl)ammonium Bromides. Energ Fuel 2013, 27:1285-1292.

22. Aman ZM, Syddall WGT, Haber A, Qin Y, Graham B, May EF, Johns ML, Pickering PF: Characterization of Crude Oils That Naturally Resist Hydrate Plug Formation. Energ Fuel 2017, 31:5806-5816.

23. Aman ZM, Haber A, Ling NNA, Thornton A, Johns ML, May EF: Effect of Brine Salinity on the Stability of Hydrate-in-Oil Dispersions and Water-in-Oil Emulsions. Energ Fuel 2015, 29:7948-7955.

24. Lauricella M, Ciccotti G, English NJ, Peters B, Meloni S: Mechanisms and Nucleation Rate of Methane Hydrate by Dynamical Nonequilibrium Molecular Dynamics. J Phys Chem C 2017, 121:24223-24234. 
25. Khurana M, Yin Z, Linga P: A Review of Clathrate Hydrate Nucleation. ACS Sustain Chem Eng 2017, 5: $11176-11203$.

26. Hawtin RW, Quigley D, Rodger PM: Gas Hydrate Nucleation and Cage Formation at a Water/Methane Interface. Phys. Chem. Chem. Phys 2008, 10: 4853-4864.

27. Jacobson LC, Molinero V: Can Amorphous Nuclei Grow Crystalline Clathrates? The Size and Crystallinity of Critical Clathrate Nuclei. J Am Chem Soc 2011, 133: 6458-6463.

28. Jimenez-Angeles F, Firoozabadi A: Nucleation of Methane Hydrates at Moderate Subcooling by Molecular Dynamics Simulations. J Phys Chem C 2014, 118:11310-11318.

29. Guo GJ, Li M, Zhang YG, Wu CH: Why can water cages adsorb aqueous methane? A potential of mean force calculation on hydrate nucleation mechanisms. Phys Chem Chem Phys 2009, 11:10427-10437.

30. Walsh MR, Rainey JD, Lafond PG, Park DH, Beckham GT, Jones MD, Lee KH, Koh CA, Sloan ED, Wu DT, et al.: The cages, dynamics, and structuring of incipient methane clathrate hydrates. Phys Chem Chem Phys 2011, 13:19951-19959

31. Hall KW, Carpendale S, Kusalik PG: Evidence from mixed hydrate nucleation for a funnel model of crystallization. P Natl Acad Sci USA 2016, 113:12041-12046.

32. Bi Y, Li T: Probing Methane Hydrate Nucleation through the Forward Flux Sampling Method. J Phys Chem B 2014, 118, 47, 13324-13332.

33. Lauricella M, Meloni S, English NJ, Peters B, Ciccotti G: Methane Clathrate Hydrate Nucleation Mechanism by Advanced Molecular Simulations. J Phys Chem C 2014, 118:22847-22857.

34. Zhang ZC, Kusalik PG, Guo GJ: Bridging solution properties to gas hydrate nucleation through guest dynamics. Phys Chem Chem Phys 2018, 20:24535-24538.

This paper employs molecular dynamics simulations to investigate nucleation of several hydrates, both sl and sll, in the presence of several guests. It shifts the focus from the activated state to the system conditions before nucleation starts, which could have important consequences in the field.

35. Poon GG, Peters B: Accelerated Nucleation Due to Trace Additives: A Fluctuating Coverage Model. J Phys Chem B 2016, 120:1679-1684.

36. Knott BC, Molinero V, Doherty MF, Peters B: Homogeneous Nucleation of Methane Hydrates: Unrealistic under Realistic Conditions. J Am Chem Soc 2012, 134:19544-19547.

37. Poon GG, Seritan S, Peters B: A design equation for low dosage additives that accelerate nucleation. Faraday Discuss 2015, 179:329-341.

38. Gobbo G, Bellucci MA, Tribello GA, Ciccotti G, Trout BL: Nucleation of Molecular Crystals Driven by Relative Information Entropy. J Chem Theory Comput 2018, 14:959-972.

A framework is introduced to enable the study of nucleation in complex system, where the order parameters traditionally used for simple systems typically do not perform well. The concept of 'relative information entropy' is employed systematically in this approach. This seems essential for studying hydrate nucleation in complex systems that contain LDHIs, a variety of compounds, and impurities.

39. Michalis VK, Tsimpanogiannis IN, Stubos AK, Economou IG: Direct phase coexistence molecular dynamics study of the phase equilibria of the ternary methane-carbon dioxide-water hydrate system. Phys Chem Chem Phys 2016, 18:23538-23548.

40. Bui T, Sicard F, Monteiro D, Lan Q, Ceglio M, Burress C, Striolo A: Antiagglomerants Affect Gas Hydrate Growth. J Phys Chem Lett 2018, 9:3491-3496.

In this manuscript two hypothesis were tested: the first is that anti-agglomerants could delay hydrate growth by forming a film impermeable to methane transport, the second is that anti-agglomerants could promote hydrate growth. Both these hypotheses were confirmed, and their effect quantified using atomistic simulations. Potentially, these insights could help design future anti-agglomerants. 
41. Ghaani MR, English NJ: Molecular Dynamics Study of Propane Hydrate Dissociation: Nonequilibrium Analysis in Externally Applied Electric Fields. J Phys Chem C 2018, 122: 7504-7515.

42. Anderson BJ, Tester JW, Borghi GP, Trout BL: Properties of inhibitors of methane hydrate formation via molecular dynamics simulations. J Am Chem Soc 2005, 127:17852-17862.

43. Jimenez-Angeles F, Firoozabadi A: Hydrophobic Hydration and the Effect of $\mathrm{NaCl}$ Salt in the Adsorption of Hydrocarbons and Surfactants on Clathrate Hydrates. Acs Central Sci 2018, 4:820-831.

44. Mehrabian H, Bellucci MA, Walsh MR, Trout BL: Effect of Salt on Antiagglomerant Surface Adsorption in Natural Gas Hydrates. J Phys Chem C 2018, 122:12839-12849.

45. Mehrabian H, Walsh MR, Trout BL: In Silico Analysis of the Effect of Alkyl Tail Length on Anti-Agglomerant Adsorption to Natural Gas Hydrates in Brine. J Phys Chem C 2019, submitted.

46. Dieker LE, Aman ZM, George NC, Sum AK, Sloan ED, Koh CA: Micromechanical Adhesion Force Measurements between Hydrate Particles in Hydrocarbon Oils and Their Modifications. Energ Fuel 2009, 23:5966-5971.

47. Bui T, Phan A, Monteiro D, Lan Q, Ceglio M, Acosta E, Krishnamurthy P, StrioloD A: Evidence of StructurePerformance Relation for Surfactants Used as Antiagglomerants for Hydrate Management. Langmuir 2017, 33:2263-2274.

48. Sicard F, Bui T, Monteiro D, Lan Q, Ceglio M, Burress C, Striolo A: Emergent Properties of Antiagglomerant Films Control Methane Transport: Implications for Hydrate Management. Langmuir 2018, 34:9701-9710.

49. Phan A, Bui T, Acosta E, Krishnamurthy P, Striolo A: Molecular mechanisms responsible for hydrate antiagglomerant performance. Phys Chem Chem Phys 2016, 18:24859-24871.

50. Khedr A, Striolo A: Quantification of Ostwald Ripening in Emulsions via Coarse-Grained Simulations. J. Chem. Theory Comput. 2019, submitted.

51. Cardellini A, Alberghini M, Rajan AG, Misra RP, Blankschtein D, Asinari P: Multi-scale approach for modeling stability, aggregation, and network formation of nanoparticles suspended in aqueous solutions. Nanoscale 2019, 11:3979-3992.

52. Apostolopoulou M, Dusterhoft R, Day R, Stamatakis M, Coppens MO, Striolo A: Estimating permeability in shales and other heterogeneous porous media: Deterministic vs. stochastic investigations. Int J Coal Geol 2019, 205:140-154.

53. Naullage PM, Bertolazzo AA, Molinero V: How Do Surfactants Control the Agglomeration of Clathrate Hydrates? ACS Central Sci 2019, 5:428-439.

These coarse-grained simulations quantify many mechanisms by which anti-agglomerants act when affecting hydrate particle agglomeration. While the study focused on non-ionic surfactants, the results seem to be generalisable, which provides a clear understanding of the complex systems involved in flow assurance.

54. Tokiwa Y, Sakamoto H, Takiue T, Aratono M, Matsubara H: Effect of Alkane Chain Length and Counterion on the Freezing Transition of Cationic Surfactant Adsorbed Film at Alkane Mixture - Water Interfaces. J Phys Chem B 2015, 119:6235-6241.

55. Tamam L, Pontoni D, Sapir Z, Yefet S, Sloutskin E, Ocko BM, Reichert H, Deutsch M: Modification of deeply buried hydrophobic interfaces by ionic surfactants. P Natl Acad Sci USA 2011, 108:5522-5525.

56. Aman ZM, Koh CA: Interfacial phenomena in gas hydrate systems. Chem Soc Rev 2016, 45:1678-1690.

57. Ma S., Sun L., Kelland M.A., Wang Q., Li D., Zhang Y., Dong J.: Hydrophobic hydration affects growth of clathrate hydrate: insight from an NMR relaxometric and calorimetric study. Chem. Commun. 2019, 55: 29362939. 


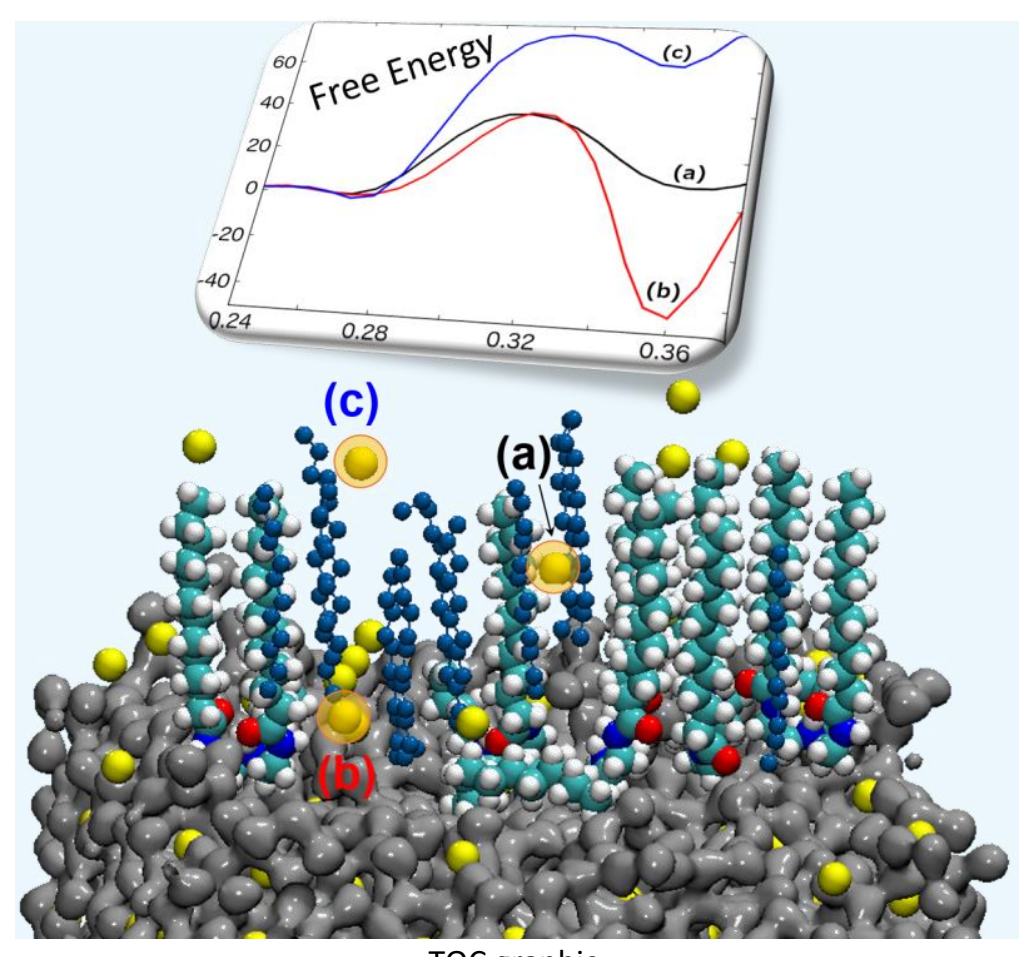

TOC graphic. 